\title{
'I think' doesn't mean 'I am'
}

It's generally thought that productive people should rise early, beginning their day with the sunrise. Then again, from childhood until the very end of his life, the French mathematician and philosopher René Descartes as a rule stayed in bed until $11 \mathrm{am}$ - and nevertheless set western philosophy on the course it followed for several hundred years. It is to Descartes that we still trace some of our deepest intuitions about mind and matter.

He brought algebra into the study of geometry, and set out methods for describing events and processes in space and time using coordinate systems. Today it is difficult to imagine physics or engineering without the language of mathematics. Descartes also helped to establish firm belief in a material world that exists 'out there', with objective properties and laws discoverable by minds working with logic and experiment (although he wasn't too keen on doing experiments himself).

Of course, he also gained lasting philosophical fame in another way - for doubting the reliability of all knowledge except for that of his own independent consciousness: "I think therefore I am". In so doing, he placed the ideal world of the mind in brute distinction to the physical world of matter, as a subjective reality that is not part of the physical world.

Ever since, students of philosophy have struggled with the contradiction between the intuitive good sense of Cartesian dualism, with distinct worlds of mind and matter, and the logical desire to somehow find a more unified picture. Can something as unique as subjective awareness ever arise from mere matter? If not, do we really believe it rests on something that is not matter?

These issues still perplex the best minds today, and in much the way that Descartes framed them. Could it be that part of the trouble lies in the way he framed them, binding us into habits of thought from which there is no way out? That's the provocative suggestion of physicist and philosopher D. M. Appleby, who argues that the Cartesian concepts of mind and matter are "like the two poles of a bar magnet, impossible to isolate", and that being locked into the framework poses an essential barrier to deeper understanding - not only of consciousness, but possibly of quantum physics and the nature of reality as well (preprint at http://arxiv.org/abs/1305.7381; 2013).

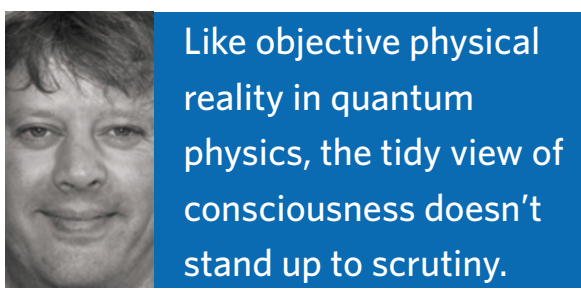

Several authors in recent years have drawn links between quantum physics and consciousness. Probably most famous was Roger Penrose, who suggested that persisting inconsistencies in the foundations of quantum theory point to as yet unknown physics, which may also play a role in the mind. This is not at all Appleby's aim. Rather, he argues that our difficulties in making sense of the weirdness of quantum physics and of consciousness have similar roots - and precisely in the philosophical categories of mind and matter that Descartes established for us.

For example, quantum physics gives us reason to be suspicious of the simple notion of an 'objective' world of matter. Even the very properties that an electron can be said to possess depend on the kind of experimental set-up an observer chooses to work with. Reality isn't just out there with properties of its own, at least not in any naive way.

"It only seems weird", as Appleby suggests, "because we insist on looking at it through Cartesian spectacles. The problem is that Cartesian assumptions have become so deeply ingrained in our thinking that it is hard to find the right non-Cartesian spectacles." That seems right to me.

So too, he suggests, with the famous 'problem of consciousness'. In our simple thinking, based on Descartes framework, the subjective mind has an awareness of an external reality. That awareness is distinct and known clearly by the self. But this view doesn't really stand up to modern neuroscience, which shows that we don't actually have access to our own conscious minds in the way we might think. For example, consider experiments that use a computer to monitor a reader's eye movements as he or she reads a page of text. The actual span of visual acuity is quite small, only one degree or so; hence, we can focus on only a couple words at a time. Suppose you rapidly replace the actual page of text with a page on which all the characters are Xs but then have the computer replace a few with real words just where, and just before, the eye next alights. In real experiments like this, readers would see the page as entirely normal. In other words, what you seem to be reading may be something else altogether.

Hence, Appleby argues, the trajectory of our conscious awareness is not as continuous as we think it is. It carries an indeterminacy that is qualitatively like that found in the trajectories of quantum systems. Like objective physical reality in quantum physics, the tidy view of consciousness doesn't stand up to scrutiny.

Curiously, Appleby notes, there was no 'ego-isolation' in philosophy before Descartes; no fixation on an isolated subjective self. As philosophers have noted, Descartes' Meditations engenders a profound alientation in its readers, leading them to experience a "problem of the external world". Why was Descartes pushed into this?

The move, Appleby suggests, was motivated as much by physics as introspection; it was an unavoidable consequence of Descartes' commitment to seeing a material world following mathematical laws as the basis of physics. If everything is seen as arising from the dynamics of things like particles and mechanical forces, then what should we make of the 'secondary' properties we know so well - things like colour, hardness or weight? Preserving these requires a complementary and subjective reality.

"The Cartesian conception of consciousness", as Appleby sees it, ends up as "a garbage can for all the many things which mathematical physicists want to be rid of."

Could breaking out of the architecture set up by Descartes be the key to understanding quantum physics - and possibly the mind as well? Abandoning 'idealism' is easy for most scientists, but Appleby's point is that we may also have to drop the notion that there is an objective mathematical description of the world. This, he notes, "produces in many people a sense of vertigo". But this only shows the absolute lock that the Galilean-Cartesian split has on our imagination. We have not yet found an alternative.

MARK BUCHANAN 